\title{
Pseudomonas syringae pv. actinidiae Effector HopAU1 Interacts with Calcium-Sensing Receptor to Activate Plant Immunity
}

\author{
Jinlong Zhang, Mingxia Zhou, Wei Liu, Jiajun Nie and Lili Huang *(D)
}

Citation: Zhang, J.; Zhou, M.; Liu, W.; Nie, J.; Huang, L. Pseudomonas syringae pv. actinidiae Effector HopAU1 Interacts with CalciumSensing Receptor to Activate Plant Immunity. Int. J. Mol. Sci. 2022, 23, 508. https://doi.org/10.3390/ ijms23010508

Academic Editor: Setsuko Komatsu

Received: 24 November 2021

Accepted: 31 December 2021

Published: 3 January 2022

Publisher's Note: MDPI stays neutral with regard to jurisdictional claims in published maps and institutional affiliations.

Copyright: (C) 2022 by the authors. Licensee MDPI, Basel, Switzerland. This article is an open access article distributed under the terms and conditions of the Creative Commons Attribution (CC BY) license (https:// creativecommons.org/licenses/by/ $4.0 /)$.
State Key Laboratory of Crop Stress Biology for Arid Areas, College of Plant Protection, Northwest A\&F University, Xianyang 712100, China; zhangjinlong04@nwsuaf.edu.cn (J.Z.); zhoumingxia@nwsuaf.edu.cn (M.Z.); wliu@nwsuaf.edu.cn (W.L.); niejiajun@nwsuaf.edu.cn (J.N.)

* Correspondence: huanglili@nwsuaf.edu.cn; Tel.: +86-029-8709-1312

\begin{abstract}
Kiwifruit canker, caused by Pseudomonas syringae pv. actinidiae (Psa), is a destructive pathogen that globally threatens the kiwifruit industry. Understanding the molecular mechanism of plant-pathogen interaction can accelerate applying resistance breeding and controlling plant diseases. All known effectors secreted by pathogens play an important role in plant-pathogen interaction. However, the effectors in $P_{s} a$ and their function mechanism remain largely unclear. Here, we successfully identified a T3SS effector HopAU1 which had no virulence contribution to Psa, but could, however, induce cell death and activate a series of immune responses by agroinfiltration in Nicotiana benthamiana, including elevated transcripts of immune-related genes, accumulation of reactive oxygen species (ROS), and callose deposition. We found that HopAU1 interacted with a calcium sensing receptor in $\mathrm{N}$. benthamiana $(\mathrm{NbCaS})$ as well as its close homologue in kiwifruit (AcCaS). More importantly, silencing CaS by RNAi in N. benthamiana greatly attenuated HopAU1triggered cell death, suggesting $\mathrm{CaS}$ is a crucial component for HopAU1 detection. Further researches showed that overexpression of $\mathrm{NbCaS}$ in $\mathrm{N}$. benthamiana significantly enhanced plant resistance against Sclerotinia sclerotiorum and Phytophthora capsici, indicating that CaS serves as a promising resistance-related gene for disease resistance breeding. We concluded that HopAU1 is an immune elicitor that targets CaS to trigger plant immunity.
\end{abstract}

Keywords: Pseudomonas syringae pv. actinidiae; effector; calcium-sensing receptor; resistance-related gene; plant immunity

\section{Introduction}

Central to plant survival is the ability to activate immunity upon pathogen perception. Plant immunity is activated by sensing either conserved microbial signatures like pathogenassociated molecular patterns (PAMPs) or specific effectors secreted by pathogens [1]. To invade successfully, pathogenic bacteria secrete type III effectors into host cells to subvert plant immunity, and in turn, plants evolve towards effector recognition for enhancing immunity. Effector-triggered immunity (ETI) through intracellular receptors interferes with pathogen invasion early in host and converts it to rapid and robust defense [2]. During the course of their co-evolution, it increases the challenge of resistance breeding for preventing disease. Resistance breeding has been demonstrated to be one of the most efficient strategies for disease management. For disease-resistance breeding, the application of resistancerelated genes is dramatically timesaving, compared with the conventional hybrids breeding approach [3]. Nonetheless, it remains a big challenge to obtain resistance-related genes before their successful application into breeding.

The exploitation of pathogen effectors to identify resistance genes is a powerful molecular tool for disease resistance breeding. Many plant resistance $(R)$ genes have been successfully detected by effector-assisted strategies in numerous plants, such as tomato, rice and Arabidopsis [4]. As one of the most studied phytopathogens, Pseudomonas syringae has been demonstrated to employ T3SS effectors (T3SEs) as its core interaction with 
plants [5-8]. However, only several have been detected by plants, subsequently activating plant immune responses [9]. For example, AvrPtoB and AvrPto can physically interact with Pto kinase and elicit plant defenses in a Pto-dependent manner [10,11]. AvrB activates the JA signaling pathway by interacting with RIN4 [12,13]. In addition, three Pseudomonas syringae T3SEs (HopX1, HopZ1a and HopBB1) have been reported to target the COI1 receptor or JAZ transcriptional repressors to activate JA signaling [12,14-16]. As well as the aforementioned well-known effectors, additional effectors exhibit the ability to activate plant immunity, yet their direct resistance targets in plants are largely unknown (e.g., HopAS1, AvrRpt2 and AvrRps4) $[17,18]$. However, due to the diverse effector repertoires among $P$. syringae pathovars [19], the recognition mechanism between pathogens and plants remains to be elucidated.

The current pandemic Pseudomonas syringae pv. actinidiae biovar 3 (Psa3), the causal agent of the kiwifruit bacterial canker, has been recorded in all major kiwifruit cultivated areas since a sudden "2008 outbreak" in central Italy, and is a serious threat to global kiwifruit production [20,21]. Psa, as one of the most aggressive air-borne bacterial diseases, can severely infect aboveground kiwifruit organs (e.g., leaves, flower buds, twigs, and young shoots), restricting the healthy and sustainable development of the global kiwifruit industry [22-25]. Despite the forest plant quarantine measures applied worldwide to prevent an epidemic outbreak, persistent control strategies are still required [26]. Currently, great efforts have been made to reveal the Psa pathogenicity, yet the corresponding T3SEs and their functional mechanism are still unclear. Studies on Psa T3SEs have performed subcellular localization and transient expression to select several T3SEs triggered HR in $N$. benthamiana [27]. In addition, $A v r E 1_{P s a}$ and $H_{o p R} 1_{P s a}$ have been shown to be essential for full virulence, while HopM1 (belonging to the AvrE/HopM family) exhibits functional redundancy [28,29]. Additional research has focused on the recognition mechanism of Psa T3SEs in model plants. HopZ $5_{P s a}$-tiggered ETI can be inhibited by SOBER1 in non-host Arabidopsis, while AvrRpm $1_{P_{s a}}$ can be recognized by Rpa1 (but not RPM1) to trigger a plant immunity response [30-33]. Other than HopZ5 and AvrRpm1, reports of Psa T3SEs that can activate plant immune responses are limited. Moreover, the resistance-related genes in kiwifruit remain largely elusive, and the identification of these genes represents a big challenge.

Herein, we aim to employ HopAU1 for screening resistance-related genes. By bioinformatics and pathogenesis analysis, we demonstrate that HopAU1 is an evolutionarily conserved effector across Pseudomonas and make no contributions to Psa virulence. Using transient expression assays we demonstrate HopAU1 can induce HR-like cell death (HCD) and trigger immune response in N. benthamiana. Further evidence reveals that HopAU1 can interact with the host and nonhost $\mathrm{CaS}$, which are responsible for HopAU1-induced cell death and, as a resistance-related gene, positively enhance plant immunity against phytopathogens in N. benthamiana. Taken together, our data reveals that HopAU1 act as an elicitor recognized by the resistance-related protein $\mathrm{CaS}$ to activate plant immunity.

\section{Results}

\subsection{HopAU1 Is an Evolutionarily Conserved Effector in Pseudomonas}

Psa was observed to be a highly diverse group in terms of the genome arrangement of both phenotypes and genotypes, including many mobile genetic elements (e.g., IME and ICE, prophages, transposons and plasmids). For the high-virulence Psa strain M228 genome, HopAU1 was identified as a single-copied gene encoding a protein of 731 amino acids and located in the plasmid with a consensus HrpL-mediated hrp box sequence in its upstream (Figure 1A). This indicates that HopAU1 as a T3SE gene may transfer among Pseudomonas syringaes via plasmids. 


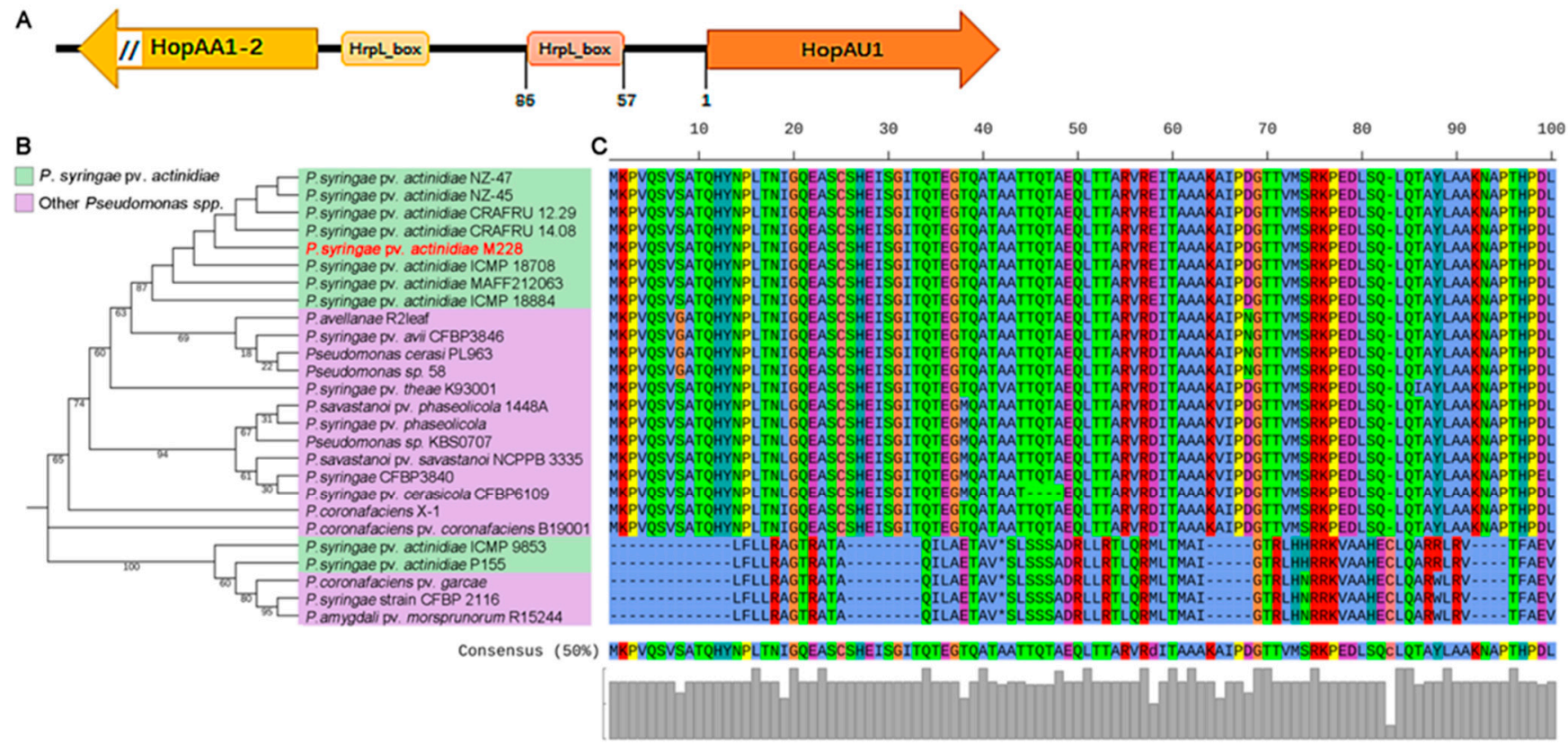

Figure 1. HopAU1 homologues are conserved and widely distributed in Pseudomonas. (A) Gene structure characteristics of HopAU1 display in plasmid genome of M228. (B) HopAU1 and its homologues nucleic acid sequences were selected according to the BLAST results against to the NCBI-Nr database. The phylogeny tree was constructed using MEGA 7.0 software with the Neighbor-joining method. The data below bootstrap column support for each branch (gaps eliminated, 1000 bootstraps); Pseudomonas syringae pv. actinidiae and other Pseudomonas spp. are marked with light blue and purple background, respectively. (C) Multiple sequence alignment of HopAU1 homologues sequences directly next to the tree visualized by iTOL annotion (100 amino acid residues as sample).

For the construction of the HopAU1 phylogenetic tree, 26 homologous sequences were selected against the National Center for Biotechnology Information (NCBI) database using the online BLASTN program. The results showed that HopAU1 was widely distributed in various Pseudomonas spp., and mainly existed in Psa clusters, forming a close clade (Figure 1B). We then investigated the protein sequence conservation of 21 homologous HopAU1 sequences by selecting 100 amino acids as a representation of multiple sequence alignments. Apart from the pre-stop translation diverse clade, 21 homologous HopAU1 sequences exhibited more than $98 \%$ similarity consisting of the full-length protein alignment (Figures 1C and S1). This suggests that HopAU1 is widely distributed and evolutionarily conserved among Pseudomonas spp.

\subsection{HopAU1 Makes No Contribution to Psa Virulence}

The relative expression level using qRT-PCR at eight different time point $(0,3,6,9,12$, 24,48 , and $96 \mathrm{~h}$ ) showed that during the early inducing stage HopAU1 was significantly upregulated compared to its relative expression at $0 \mathrm{~h}$. HopAU1 was significantly up-regulated at 3, 6, 9 and $12 \mathrm{~h}$, and its highest expression was observed at $9 \mathrm{~h}$ (fold change $>50$ ) (Figure 2A). The expression pattern of HopAU1 indicated is possible functionality during early infection.

In order to explore the virulence contribution of HopAU1 in M228, we successfully obtained HopAU1-deletion mutant based on the homologous recombination method (Figure S2A). The virulence assays of the lesion areas did not reveal any significant differences between M228 and $\triangle H o p A U 1$ on kiwifruit leaf discs (Figure 2B). This was also true for the kiwifruit canes (Figure $2 \mathrm{C}$ ). In order to facilitate further analysis of the HopAU1 function in Psa, we constructed a 28 T3SEs-deletion mutant containing HopAU1 in M228 denoted as $\mathrm{M} 228 \Delta 28 \mathrm{E}$ (unpublished). The single HopAU1-complement mutant M228 $28 \mathrm{E}-\mathrm{C}-H o p A U 1$ was then adopted to evaluate its virulence (Figure S2A,B). The virulence test assays did not 


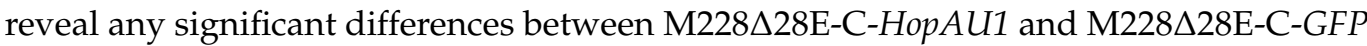
on the kiwifruit leaf discs or canes (Figure S3A,B). These results demonstrate the limited influence of HopAU1 on the kiwifruit leaf discs and canes.

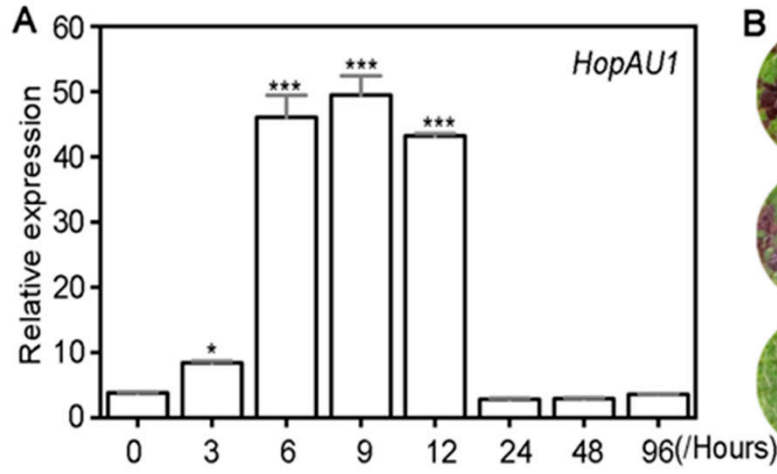

C

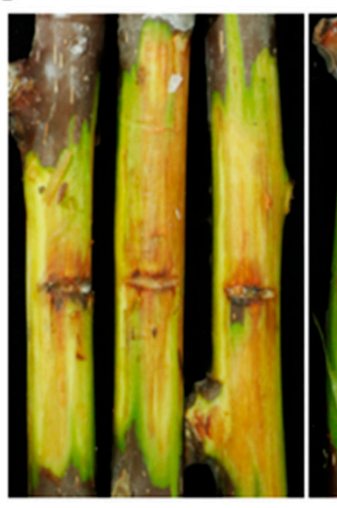

M228

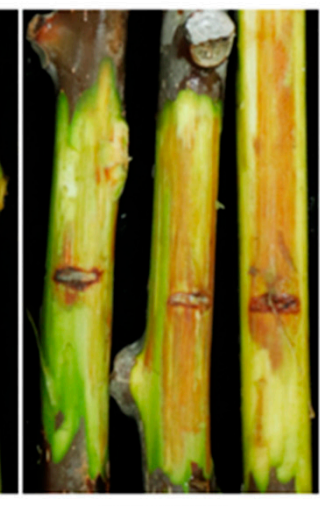

$\triangle H o p A U 1$
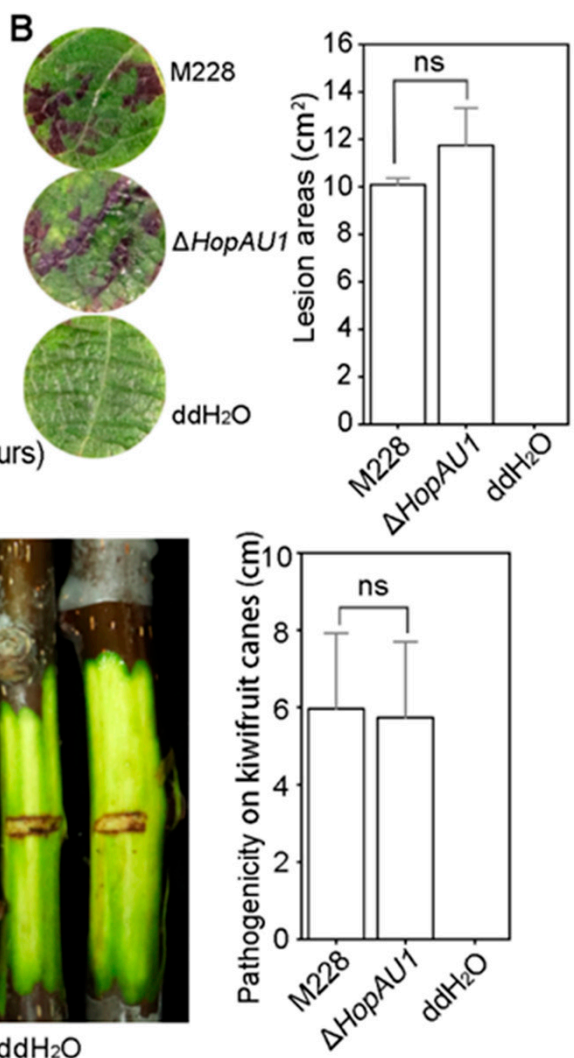

Figure 2. HopAU1 shows no obvious contribution to $P_{s a}$ M228 virulence on kiwifruit leaf dises and canes. (A), Relative expression level of HopAU1 in hrp-inducing media at 0, 3, 6, 12, 24, 48, and $96 \mathrm{~h}$. The experiment repeats twice with similar results. (B), No significant differences in virulence were detected between M228 and $\triangle H o p A U 1$ post-inoculation on leaf discs of Actinidiae chinensis cv. HongYang. (C), No significant differences in virulence were detected between M228 and $\Delta$ hopAU1 post-inoculation on kiwifruit canes of Actinidiae chinensis cv. 'HongYang'. The symptoms of B and $\mathrm{C}$ were observed at 5 days and 20 days post inoculation, respectively. For the inoculation, at least 15 canes or leaf discs were used for each treatment with three independent biological experiments. The statistical data were visualized with Mean \pm standard errors (SEs) using Student's $t$-test $(* * *, p<0.001$; *, $p<0.05 ;$ ns, no significant, $p>0.05$ ).

\subsection{Transient Expression of HopAU1 Triggers Cell Death in N. benthamiana}

The transient expression of HopAU1 in N. benthamiana showed that HopAU1 triggered cell death after 3 days post-Agro-infiltration together with Bax. However, this was not true for the control GFP. The successful expression of HopAU1 was further confirmed by western blotting. In addition, electrolyte leakage assays also demonstrated significant differences between HopAU1 and GFP (Figure 3A-C). Therefore, HopAU1 can induce HR-like cell death (HCD) in N. benthamiana.

\subsection{HopAU1 Induces Immune Responses in N. benthamiana}

The aforementioned HopAU1 transiently expression in N. benthamiana leaves was sufficient to induce HR-like cell death 3 days post agroinfiltration. To test whether HopAU1induced cell death had a relationship with plant immunity response, we examined the accumulation of reactive oxygen species (ROS) burst and callose deposition, and the transcript accumulation levels of two corresponding signal pathway marker genes, HSR203J 
and HIN1 [34,35], in N. benthamiana. Figure 4A showed that ROS and callose were massive accumulated in $N$. benthamiana leaves after 2 days infiltration. Meanwhile, transcript accumulation levels of HSR203J and HIN1 were significant up-regulated about 37-fold and 8 -fold, respectively (Figure 4B). Those similar results indicate that HopAU1 was recognized by $N$. benthamiana and -triggered cell death related to plant HR immune response.

A

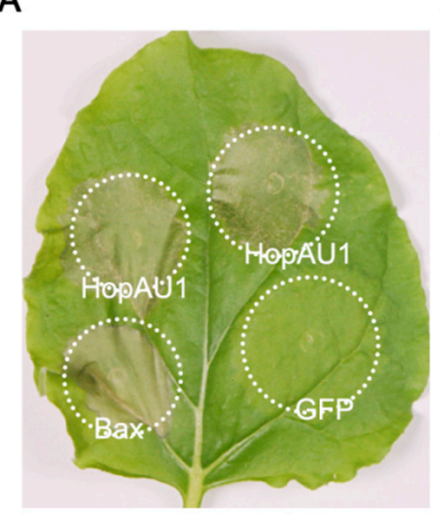

B

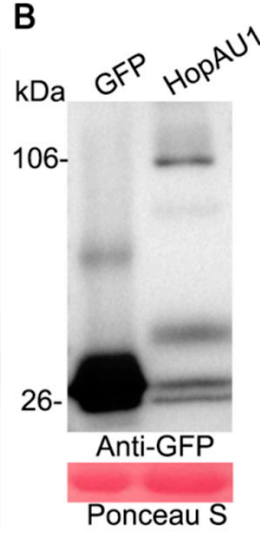

C

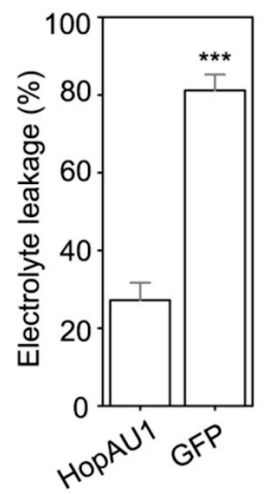

Figure 3. HopAU1 induces cell death in Nicotiana benthamiana. (A) HopAU1-induced cell death in N. benthamiana. The cell death phenomenon was photographed after 3 days in leaves of $N$. benthamiana with Agrobacterium tumefaciens carrying pCAMBIA1302-HopAU1-GFP as treatment, pCAMBIA1302Bax and pCAMBIA1302-GFP as positive and negative control, respectively. (B) Western blotting analysis of proteins of $N$. benthamiana by agroinfiltration mediated transiently expression green fluorescent protein (GFP) control and HopAU1 tagged GFP. (C) Quantification of cell death by measuring electrolyte leakage after 3 days. The statistical data represent means \pm standard errors (SEs) with three independent biological experiments; asterisks show significant differences between selected and EV for that assessment ${ }^{* * *}, p<0.001$; Student's $t$-test).
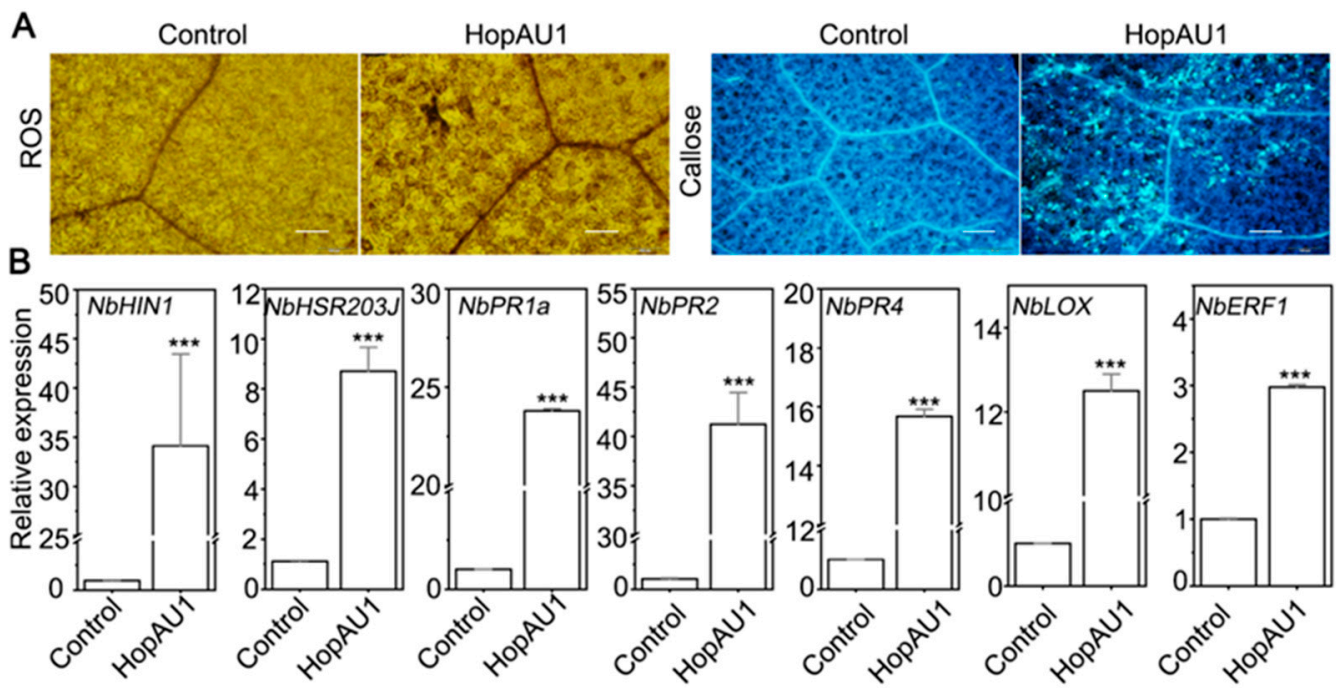

Figure 4. HopAU1 activates immunity responses in Nicotiana benthamiana. (A) ROS accumulation and callose deposition in N. benthamiana leaves by transiently expressing HopAU1-GFP and GFP control, respectively; Photographs were taken 2 days after infiltration; Bars $=50 \mu \mathrm{m}$. (B) qRT-PCR analysis of hypersensitive-response-speicfic marker genes and defense-related marker gene in $N$. benthamina. Transcript levels of candidate genes were normalized to that of NbActin and GFP was used as control. The statistical data were visualized with Mean \pm standard errors (SEs) using Student's $t$-test $\left.{ }^{* * *}, p<0.001\right)$. 
In order to further investigate whether HopAU1 expression altered plant immune pathways, we detected the transcript accumulation levels of well-validated immunerelated marker genes in N. benthamiana, including two vital marker genes of salicylic acid (SA)-dependent immunity, NbPR1 $a$ and NbPR2 [36]; two marker genes of jasmonic acid (JA)-dependent immunity, $N b P R 4$ and $N b L O X[37,38]$; and one marker gene of ethylenedependent, NbERF1 [37,39]. As showed in Figure 4B, the relative expression levels of all selected immunity-related marker genes were up-regulated with varying degrees when transiently expression HopAU1 after 2 days post agroinfiltration. These results demonstrate that HopAU1 acts as an elicitor that induces defense response in N. benthamiana.

\subsection{HopAU1 Interacts with Plant Calcium Sensing Receptor (CaS)}

In order to clarify the underlying mechanism between HopAU1 and the plant, we employed immunoprecipitation-mass spectrometry (IP-MS) to screen its candidate targets. In particular, HopAU1-GFP fusion protein was transiently expressed in N. benthamiana for $48 \mathrm{~h}$ and the bound proteins were then collected via GFP-Trap Immunomagnetic beads for IP-MS analysis. A total of 22 candidate targets were selected to identify the interaction relationship with HopAU1 (Supplementary Table S2). Large scale screening via by bimolecular fluorescent complementation (BiFC) and co-immunoprecipitation (CoIP) revealed that HopAU1 interacted with a calcium sensing receptor $(\mathrm{CaS})$ in $N$. benthamiana (Figure 5A,B). In addition, the homologous protein AcCaS in kiwifruit could also positively interact with HopAU1 by CoIP (Figure S4). These results demonstrate the in-vivo and in-vitro interactions between HopAU1 and CaS.
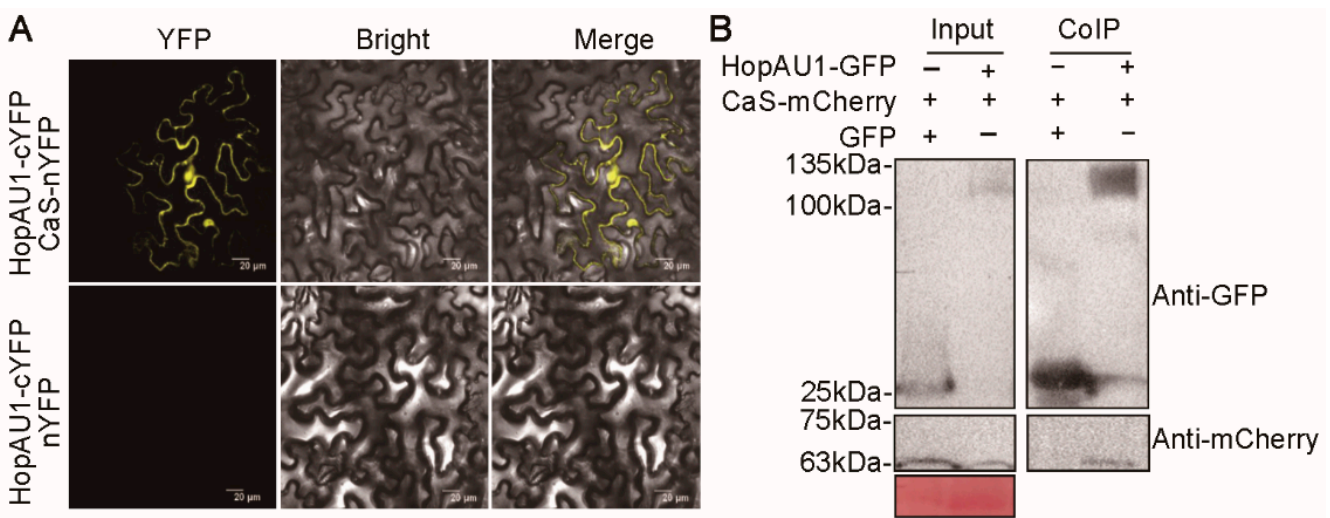

Figure 5. Confirmation of the interaction between HopAU1 and CaS. (A) BiFc analysis of HopAU1 and CaS in N. benthamiana. Photographs were taken after 2 days post HopAU1 tagged cYFP and CaS tagged nYFP co-expression in N. benthamiana leaves. Bars $=20 \mu \mathrm{m}$. (B) Co-immunoprecipitation (CoIP) was performed for confirming the interaction. HopAU1-GFP and CaS-mCherry (or GFP and CasmCherry) were transiently co-expressed in N. benthamiana. Total protein was extracted and western blotting with anti-GFP or anti-mCherry antibodies, respectively. The bound proteins were affinity purified by GFP-Trap Immunomagnetic beads and then detected with the indicated antibodies.

\subsection{CaS Is Required for HopAU1-Triggered Cell Death in N. benthamiana}

To further determine whether CaS was required for HopAU1-triggered cell death, we generated VIGS (virus-induced gene silencing, VIGS) constructs to target the CaS expression in $N$. benthamiana. At 3 weeks after the VIGS treatments, the plants exhibited etiolation and dwarf phenotypes, and were then agroinfiltrated with GFP or HopAU1 (Figure S5). qRT-PCR analysis revealed the efficiency of CaS-silenced to fall to almost 95\% compared with the control (Figure 6D). HopAU1 failed to trigger cell death in CaSsilenced $N$. benthamiana, yet it could trigger cell death in the control (Figure 6A,B). In addition, western blotting revealed that the transient expression of HopAU1 and GFP were successful in the corresponding silenced plants (Figure 6C). These results indicate CaS to be responsible for HopAU1-induced cell death in N. benthamiana. 
A
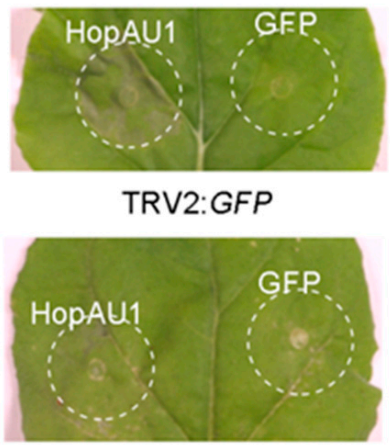

TRV2:CaS

C

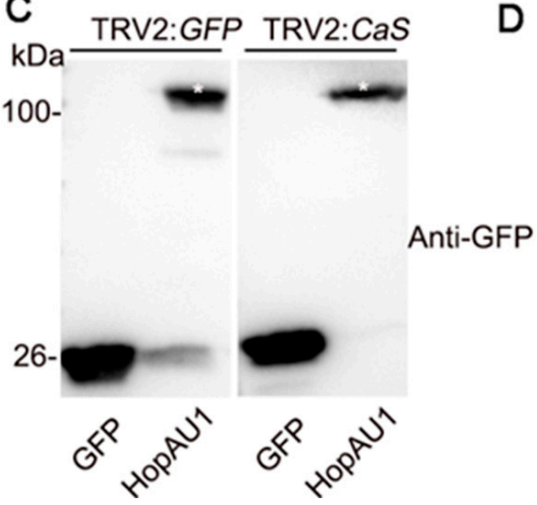

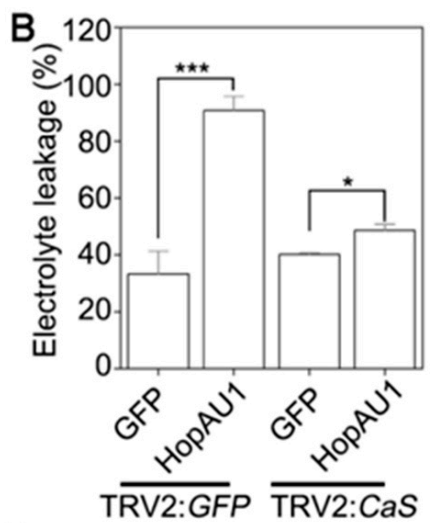

D

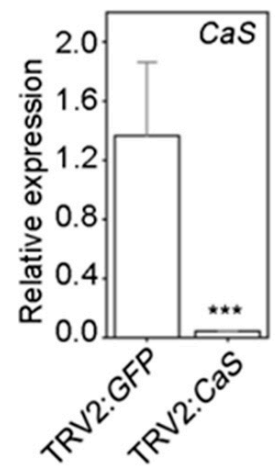

Figure 6. The CaS-silencing compromise HopAU1-induced cell death in Nicotiana benthamiana. (A) GV3101 containing HopAU1-GFP and GFP constructs were infiltrated into the region of the silenced N. benthamiana leaves. Cell death was recorded 3 days post infiltration. (B) Quantification of cell death was measured by electrolyte leakage. (C) HopAU1-GFP and GFP were transiently expressed in TRV-mediated silenced plant leaves. Immunoblot analysis the bounded proteins 2 days post inoculation. (D) The silencing efficiency was analyzed by qRT-PCR and photographs were taken at 3 weeks after viral inoculation. The Nbactin was used as the internal reference gene. Three biological repeats were performed in all experiments. Statistical data were visualized with Mean \pm standard errors (SEs) using Student's t-test $\left({ }^{* * *}, p<0.001 ;{ }^{*}, p<0.05\right)$.

\subsection{Overexpression of CaS Mediates N. benthamiana Resistance to Sclerotinia Sclerotiorum and} Phytophthora Capsici

In order to further understand the CaS-influenced plant resistance, we generated the target protein $\mathrm{CaS}$ over-expression construct and inoculated a fungal pathogen and oomycete 3 days post-inoculation on the corresponding transient over-expression of N. benthamiana in leaves. The lesion areas were observed to be significantly attenuated following infection with the fungus $S$. sclerotiorum and the oomycete pathogen $P$. capsici, compared with the control (Figure 7A-D). This indicates the ability of CaS to enhance plant resistance against filamentous pathogen infection. 

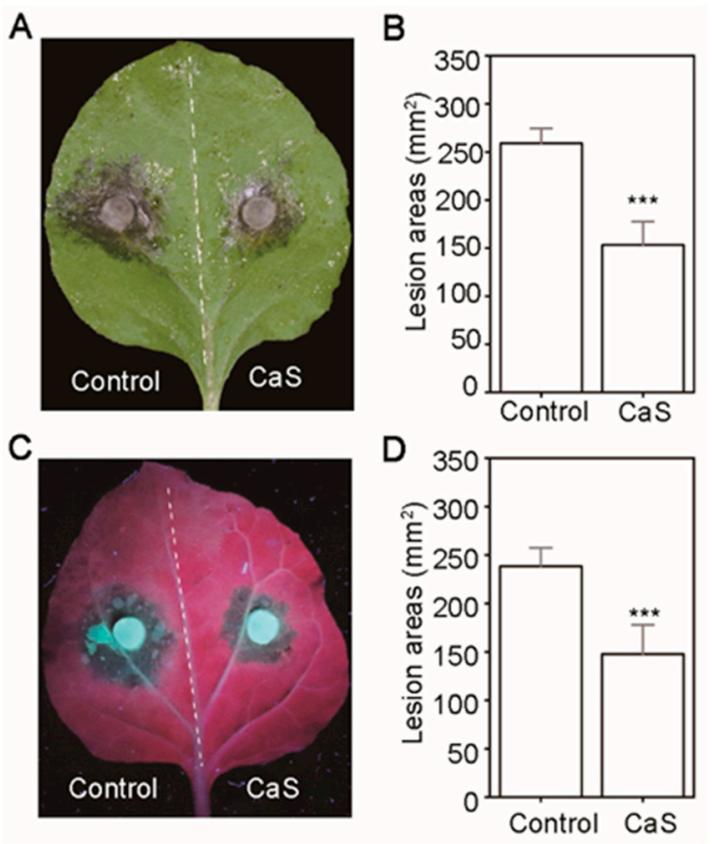

Figure 7. Overexpressing CaS enhances N. benthamiana resistance to Sclerotinia sclerotiorum and Phytophthora capsici. (A) N. benthamiana leaves were transiently expressed GFP (Left, control) and HopAU1-GFP (Right) 2 days before pathogen inoculation. Cell death was recorded at $24 \mathrm{~h}$ after inoculation of S. sclerotiorum. (C) Symptom of $N$. benthamiana leaves were photographed at $60 \mathrm{~h}$ after inoculation of P. capsici. (B,D) Lesion areas of $N$. benthamiana infected by S. sclerotiorum and P. capsici respectively, were calculated by ImageJ program. The experiment was conducted three biological repeats with at least ten technical replicates each and concluded with similar results. The statistical data was represented by Mean \pm standard errors (SEs) using Student's $t$-test $\left.{ }^{* * *}, p<0.001\right)$.

\section{Discussion}

The current pandemic Pseudomonas syringae pv. actinidiae biovar 3 (Psa3) has been identified as the most destructive disease to the global kiwifruit industry since 2008, causing bacterial organ diseases and cell deaths [20,21,40]. Thus far, only the monomorphic population $P_{s a} 3$ has been reported in China, although it was known as divergence seriously, and biodiversity and genetic variation of population in destination countries [41-43]. This new aggressive phytopathogen directly delivers abundant T3SEs to interfere with host cellular processes and facilitate invasion. However, it is not clear how the T3SEs interacted with each other on the molecular level. Here, we identified a T3SE protein HopAU1, which could be recognized by the plants, and was conserved and widely distributed in Pseudomonas spp. However, the underlying interaction between HopAU1 and the plants was not clear.

HopAU1 is a plasmid-born protein. We predicted that HopAU1 was transferred from the mobile genetic element-namely, the plasmid-bringing new features to the Psa. In addition, a classical $h r p$ box sequence was observed in the upstream of the HopAU1 gene, targeted by the global regulator HrpL (Figure 1A), which was the key component of the HrpR/S-HrpL-T3SS/T3SEs hierarchical regulatory cascade in P. syringae. To further verify the HopAU1 function in M228, we tested the virulence of $\triangle H o p A U 1$ and M228 $28 \mathrm{E}-\mathrm{C}$ HopAU1 via their inoculation on kiwifruit leaves and canes. The mutant pathogenicity did not exhibit any significant changes compared to the control, indicating that HopAU1 did not contribute to $P s a$ virulence (Figure 2B,C and Figure S3A,B). This may be attributed to the possible role of HopAU1 as a no virulence protein in the M228 T3SE repertoire. More evidences showed that three T3SEs, HopR1, AvrE1 and HopZ5, contributed almost $80 \%$ to $P s a 3$ virulence [28,41], further supporting the no-virulence protein role of in Psa.

Furthermore, we used the $A$. tumefaciens-mediated transiently expression to examine the biological function of HopAU1 in N. benthamiana. In this study, HopAU1 was able 
to induce HR-like cell death and immunity responses, suggesting it could be recognized by the plants (Figure 3A,B). We then identified CaS from N. benthamiana or kiwifruit as the target protein interacting with HopAU1 in-vivo and in-vitro. Based on bioinformatics analysis, we determined the CaS gene to be located in the nucleus chromosome, which possessed a chloroplast transit peptide, indicating that CaS transcription occurred in nuclear, processed from Endoplasmic reticulum to Golgi apparatus, and eventually ended up in the chloroplast. Chloroplasts play an important role in the defense response plants and are a common target of the phytopathogen effectors [44]. HopI1 located in chloroplasts from $P$. syringae suppresses SA accumulation and leads to the reconstruction of the thylakoid structure [45]. HopN1, also from P. syringae, targets the chloroplasts PsbQ, suppressing the defense-associated ROS burst [46]. Moreover, the fungal and viral effectors target the chloroplasts to inhibit host resistance $[47,48]$. Our results suggest that HopAU1 triggers the immune response through interactions with $\mathrm{CaS}$ and subsequently affects the biological function of CaS. As we expected, the HopAU1 transient expression in CaS-silenced N. benthamiana attenuated the cell death degree (Figure $6 \mathrm{~A}, \mathrm{~B}$ ). This indicates that CaS was specifically required for the HopAU1-triggered cell death. The CaS-mediated resistance response is associated with $\mathrm{Ca}^{2+}$ signaling which is able to cause programmed cell death (PCD) $[47,48]$.Thus, the interaction between HopAU1 and CaS may assist CaS perception and transmission of calcium signaling eventually promoting plant cell death.

$\mathrm{CaS}$ is a $\mathrm{Ca}^{2+}$ sensing receptor involving modulation cytoplasmic $\mathrm{Ca}^{2+}$ concentrations and the regulation of the stomatal closure. However, the fine molecular function of $\mathrm{CaS}$ is unclear [49-53]. Previous studies have also demonstrated that $\mathrm{CaS}$ is required for defensive hormonal responses and anti-pathogens resistance [54,55]. For example, CaS positively regulates SA accumulation, ROS bursts, nuclear-encoded defense gene transcriptional reprogramming, and is required for PTI-induced and R gene-mediated ETI resistance [48]. Moreover, previous research has revealed that plant pathogens (e.g., the fungus S. sclerotiorum and the virus Geminivirus from different kingdoms) inhibit SA accumulation to facilitate infections by interacting with $\mathrm{CaS}[47,48,55]$. Similar results in our study showed that the CaS-overexpression in N. benthamiana leaves restricted infection by filamentous pathogens compared with the control (Figure 7A-D), indicating $\mathrm{CaS}$ as a positive regulator of plant immunity to against the pathogens. These results further highlight the potential of $\mathrm{CaS}$ as a promising resistance-related protein in plant immunity responses.

\section{Materials and Methods}

\subsection{Strains and the Growth of Plants}

The Psa strain M228 was isolated from Mei County of Shaanxi province from infected leaves of Actinidia chinensis cv. Hongyang. The bacteria were grown in Luria-Bertani (LB) at $37^{\circ} \mathrm{C}$ for Escherichia coli strain, $28^{\circ} \mathrm{C}$ for Agrobacterium tumefaciens strain GV3101, and $25^{\circ} \mathrm{C}$ for Pseudomonas syringae pv. actinidiae ( $P s a$ ) containing appropriate antibiotics. All bacterial strains were stored in $25 \%$ glycerol solution at $-80{ }^{\circ} \mathrm{C}$. N. benthamiana seedlings were growing in a long-day glasshouse $\left(14 \mathrm{~h} 22^{\circ} \mathrm{C}: 10 \mathrm{~h} 20^{\circ} \mathrm{C}\right.$, day:night, $70 \%$ relative humidity).

\subsection{Plasmid Construction}

For Immunoblotting and confocal microscopy assays, HopAU1 fragments were amplified from M228 genome, and CaS fragments from $N$. benthamiana cDNA and kiwifruit cDNA, respectively (gene-specific primers listed in Supplementary Table S1). Then the obtained gene fragments were separately ligated into pCAMBIA1302-GFP and PICHmCherry (SpeI and NcoI for pCAMBIA1302-GFP, and ClaI and SpeI for PICH-mCherry), using ClonExpress II One-Step Cloning Kit (Vazyme, Nanjing, China). For constructing HopAU1-deletion recombinant vector, upstream and downstream flank of target genes separately amplified by PCR from M228 genome, and then cloned into the digested site of the suicide vector $\mathrm{pK} 18 m o b s a c B$ (The upstream and downstream flank sequence length is about $700 \mathrm{bp}-1000 \mathrm{bp}$, EcoRI and HindIII for pK18mobsacB). For obtaining HopAU1-complement recombinant vector, HopAU1 with a C-terminally tagged VSV-G was cloned into the di- 
gested site of the expression vector pDSK-GFPuv (NdeI and BamHI for pDSK-GFPuv) [56]. All constructs were confirmed by Sanger sequencing (Sangon Biotech, Shanghai, China). All mentioned primers in this study were listed in Supplementary Table S1.

\subsection{Gene-Deletion and -Complement Mutants Generation}

Gene-deletion mutants were based on the $S a c B$-unmarked homologous replacement methods [57]. For obtaining HopAU1-deletion mutant of M228, the pK18mobsacB recombinant constructs were transferred from $E$. coli strain S17-1/ $\lambda$ pir into M228 by conjugation transfer on LB agar for 2 days. Then, the transconjugants were selected on LB agar with appropriate antibiotics (nalidixic acid, $10 \mathrm{mg} / \mathrm{L}$; ampicillin $10 \mathrm{mg} / \mathrm{L}$; kanamycin, $50 \mathrm{mg} / \mathrm{L}$ ) and confirmed by PCR (using primers SacB-F/R for the vectors and $P s a-F / R$ for $P s a$ stains). Next, the conjugants were performed to a counter-selection integration on $15 \%$ sucrose LB agar. Finally, the positive mutants were validated by PCR using detecting primer pairs (Supplementary Table S1). Following the SacB-based in-frame deletion method described above, M228 $28 \mathrm{E}$ (unpublished, 28 T3SE genes were knocked out in M228 including HopAU1) and $\triangle$ HopAU1 (single HopAU1-deletion in M228) mutants were finally obtained by largely screening (Figure S2A).

For obtaining complement mutants, the empty vector pDSK-GFPuv and carrying HopAU1 were transformed into M228 $\triangle 28 \mathrm{E}$ by electroporation, respectively. Then, the

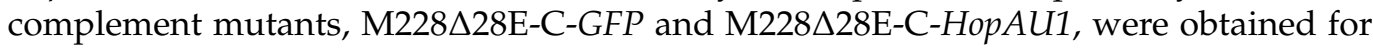
further pathogenicity analysis, and confirmed by Sanger sequencing (Figure S2B).

\subsection{Bacterial Infiltrations for HR and Electrolyte Leakage Assays in N. benthamiana}

The recombinant constructs were transformed into A. tumefaciens strain GV3101 by electroporation. For transient expression, A. tumefaciens strain GV3101 carrying HopAU1 was cultured in Luria-Bertani (LB) at $28{ }^{\circ} \mathrm{C}$ for $48 \mathrm{~h}$, and then pelleted by centrifugation and resuspended in MES buffer (10 mM magnesium chloride $\left(\mathrm{MgCl}_{2}\right), 10 \mathrm{mM}$ MES, $200 \mu \mathrm{M}$ acetosyringone, $\mathrm{pH}$ 5.7) in the dark for $3 \mathrm{~h}$ at room temperature (RT). Then, the 4-week-old $N$. benthamiana leaves was syringe infiltrated with $A$. tumefaciens strain adjusted to $4 \times 10^{8} \mathrm{cfu} / \mathrm{mL}$. Symptoms were evaluated by electrolyte leakage as described [58] and photographed after 3 days. GFP and Bax were used as negative and positive controls, respectively. All of the above experiments were repeated three times and produced similar results.

\subsection{RNA Isolation, cDNA Synthesis, and Expression Analysis}

Bacterial RNA extraction: The bacteria were grown in $\mathrm{LB}$ at $25^{\circ} \mathrm{C}$ to $\mathrm{OD}_{600}$ of 0.2 , harvested and washed three times adjust to $\mathrm{OD}_{600}$ of 0.5 . Then the bacteria were transformed into $h r p$-derepressing minimal medium (HDM) for inducing expression by a ratio of 1:40. Samples were harvested, and total RNA of bacteria was extracted using RNApure Bacteria Kit (CWBIO, Jiangsu, China) according to the manufacturer's protocol.

Plant RNA extraction: $N$. benthamiana leaves (4-week-old) were syringe infiltrated with bacterial strains. The infected leaves were sampled, frozen in liquid nitrogen and ground into power for RNA extraction. The total RNA was extracted by Quick RNA isolation Kit (Huayueyang, Beijing, China) following the manufacturer's instructions.

All related cDNA were synthesized with 1 ng of total RNA in a $20 \mu \mathrm{L}$ reverse transcription mix (Thermo Scientific, Waltham, MA, USA) for next qRT-PCR assays on CFX connect Real-time System (Bio-Rad, Hercules, CA, USA). The gyrB $[59,60]$ and the NbActin gene [61] was used as reference gene in $P s a$ and $N$. benthamiana respectively. The relative expression level used was the $2^{-\Delta \Delta C T}$ method, as described [62].

\subsection{Confocal Microscopy and Co-Immunoprecipitation Assay (CoIP)}

For BiFC assays, HopAU1 and $\mathrm{CaS}$ were cloned into vector pBin61 forming recombination constructs (HopAU1-cYFP and nYFP-CaS) and then separately transformed into $A$. tumefaciens strain GV3101 by electroporation. The infected N. benthamiana leaves was pho- 
tographed 3 days post-infiltration using a confocal laser scanning microscope (Olympus microscope FV1000, Tokyo, Japan) (YFP: excitation/emission wavelengths of $514 \mathrm{~nm} / 530 \mathrm{~nm}$ $575 \mathrm{~nm})$.

For CoIP assays, HopAU1-GFP and CaS-mCherry were transiently co-expressed in $N$. benthamiana and then were sampled for total protein extraction after 2 days. The same amounts of total proteins were incubated with pre-treated anti-GFP magnetic beads (ChromoTek $\mathrm{GmbH}$, Munich, Germany) for $2 \mathrm{~h}$ at $4{ }^{\circ} \mathrm{C}$ according to the manufacturer's instructions. Immunoprecipitates were analyzed by western blotting as described previously [63].

\subsection{Callose and Reactive Oxygen Species (ROS) Staining}

$N$. benthamiana leaves (4-week-old) were infiltrated with A. tumefaciens carrying recombinant constructs for transient expression. For callose staining, six leaf disks $(1 \mathrm{~cm}$ diameter) were decolored by heating with $95 \%$ alcohol and then were placed in trypan blue liquid (0.01\% trypan blue, $\left.150 \mathrm{mM} \mathrm{K}_{2} \mathrm{HPO}_{4}, \mathrm{pH} 9.5\right)$ for $12 \mathrm{~h}$ overnight as described [64]. For $\mathrm{H}_{2} \mathrm{O}_{2}$ staining, the equated sampled were stained on diaminobezidin (DAB) liquid ( $1 \mathrm{mg} / \mathrm{mL}$ DAB, pH 3.8) at $12 \mathrm{~h}$ light and then decolored in 95\% ethanol. All stained samples were observed and photographed using a confocal microscope (Olympus microscope FV1000, Tokyo, Japan). The experiments were repeated at least three times.

\subsection{VIGS in N. benthamiana}

For gene silencing assays, two lower leaves of two-week-old $N$. benthamiana were infiltrated with $A$. tumefaciens strain GV3101 containing pTRV1 and pTRV2 constructs. The infected plants were grown for 3 weeks before $A$. tumefaciens infection and HR assays. pTRV2:PDS and TRV2:GFP were used as controls and gene silencing level was confirmed by qRT-PCR.

\subsection{Pathogenicity Tests}

According to two indoor bioassay methods as previous described [41], vacuum infiltration and wound inoculation were used for pathogenicity tests. In experiments, at least fifteen kiwifruit leaf discs and canes were carried out for pathogenicity tests, and a replicated mock inoculation with sterile water was included as a negative control. The inoculated leaf discs and canes were incubated in a climate chamber at $16 \mathrm{~h} 18{ }^{\circ} \mathrm{C}: 8 \mathrm{~h} 14^{\circ} \mathrm{C}$, day:night, 75\% relative humidity. Analyses were repeated three times.

\subsection{Accession Numbers and Bioinformatics Analysis}

Sequence data accession nos. in this article was mentioned as follows. HopAU1 $1_{\mathrm{M} 228}$ (CN228_RS32770, https:/ / www.ncbi.nlm.nih.gov /, accessed on 18 February 2021), NbCaS (Niben101Scf18639g00026.1, https://solgenomics.net/, accessed on 6 April 2021), and AcCaS (Achn344161, http:/ / kiwifruitgenome.org/, accessed on 6 April 2021).

For phylogenetic analysis, the HopAU1 homologous sequences of different species were obtained from NCBI database by querying HopAU1 nucleic acid sequence against Non-Redundant Protein/Nucleotide Sequence Database (Nr/nt), using the online BLASTN program (https:/ / blast.ncbi.nlm.nih.gov/Blast.cgi, accessed on 18 February 2021), with default settings. Conserved domains of HopAU1 were predicted by querying protein sequence against CDD v3.19-58,235 PSSMs database, using Batch CD-Search program (https: / / www.ncbi.nlm.nih.gov / cdd, accessed on 18 February 2021). The phylogenetic tree was constructed by the Mega7.0 with Neighbor-joining method, and beautified with tree annotation, colored ranges and multiple sequence alignments, by iTOL online website (https:/ /itol.embl.de/itol_account.cgi, accessed on 15 August 2021).

\section{Conclusions}

Taken together, our results identify a conserved T3SE HopAU1 distributing across various Pseudomonas spp., which can trigger plant immunity response. More studies 
reveal the interaction between HopAU1 and CaS in N. benthamiana or kiwifruit. Here, we successfully employ the effector HopAU1 to screen resistance-related protein CaS, as a resistance resource, which is expected to be used for molecular resistance breeding against plant pathogens in the future.

Supplementary Materials: The following supporting information can be downloaded at: https: //www.mdpi.com/article/10.3390/ijms23010508/s1.

Author Contributions: J.Z., J.N. and L.H. designed the research. J.Z. and M.Z. performed the experiments. J.Z. and M.Z. analyzed the data. J.Z., J.N. and W.L. wrote the manuscript. All authors contributed to the article and approved the submitted version. All authors have read and agreed to the published version of the manuscript.

Funding: This work was financially supported by the Special Support Plan for High-level Talent of Shaanxi Province (to L.H.), the Natural Science Foundation of China (Grant no.32102174, to W.L.), and First-class Universities and Academic Programs of Northwest A\&F University (to L.H.).

Institutional Review Board Statement: Not applicable.

Informed Consent Statement: Not applicable.

Data Availability Statement: All data supporting the findings of this study are provided in the manuscript and its Supplementary Files. Additional data supporting the findings of this study are available from the corresponding authors upon request.

Acknowledgments: We thank Associate Professor Zhi-Bo Zhao at Guizhou University for helpful suggestions to improve this article.

Conflicts of Interest: The authors declare no conflict of interest.

\section{References}

1. Jones, J.D.G.; Dangl, J.L. The plant immune system. Nature 2006, 444, 323-329. [CrossRef] [PubMed]

2. Cui, H.; Tsuda, K.; Parker, J.E. Effector-triggered immunity: From pathogen perception to robust defense. Annu. Rev. Plant Biol. 2015, 66, 487-511. [CrossRef] [PubMed]

3. Breseghello, F.; Coelho, A.S.G. Traditional and modern plant breeding methods with examples in rice (Oryza sativa L.). J. Agric. Food Chem. 2013, 61, 8277-8286. [CrossRef]

4. Hammond-Kosack, K.E.; Parker, J.E. Deciphering plant-pathogen communication: Fresh perspectives for molecular resistance breeding. Curr. Opin. Biotech. 2003, 14, 177-193. [CrossRef]

5. Stavrinides, J.; McCann, H.C.; Guttman, D.S. Host-pathogen interplay and the evolution of bacterial effectors. Cell. Microbiol. 2008, 10, 285-292. [CrossRef] [PubMed]

6. Lindeberg, M.; Cunnac, S.; Collmer, A. The evolution of Pseudomonas syringae host specificity and type III effector repertoires. Mol. Plant Pathol. 2009, 10, 767-775. [CrossRef] [PubMed]

7. Khan, M.; Seto, D.; Subramaniam, R.; Desveaux, D. Oh, the places they'll go! A survey of phytopathogen effectors and their host targets. Plant J. 2018, 93, 651-663. [CrossRef] [PubMed]

8. Dillon, M.M.; Almeida, R.N.D.; Laflamme, B.; Martel, A.; Weir, B.S.; Desveaux, D.; Guttman, D.S. Molecular evolution of Pseudomonas syringae Type III secreted effector proteins. Front. Plant Sci. 2019, 10, 418. [CrossRef]

9. Laflamme, B.; Dillon, M.M.; Martel, A.; Almeida, R.N.D.; Desveaux, D.; Guttman, D.S. The pan-genome effector-triggered immunity landscape of a host-pathogen interaction. Science 2020, 367, 763-768. [CrossRef] [PubMed]

10. Kim, Y.J.; Lin, N.C.; Martin, G.B. Two distinct Pseudomonas effector proteins interact with the Pto kinase and activate plant immunity. Cell 2002, 109, 589-598. [CrossRef]

11. Luo, Y.; Caldwell, K.S.; Wroblewski, T.; Wright, M.E.; Michelmore, R.W. Proteolysis of a negative regulator of innate immunity is dependent on resistance genes in Tomato and Nicotiana benthamiana and induced by multiple bacterial effectors. Plant Cell 2009, 21, 2458-2472. [CrossRef]

12. Cui, H.T.; Wang, Y.J.; Xue, L.; Chu, J.F.; Yan, C.Y.; Fu, J.H.; Chen, M.S.; Innes, R.W.; Zhou, J.M. Pseudomonas syringae effector protein AvrB perturbs Arabidopsis hormone signaling by activating MAP Kinase 4. Cell Host Microbe 2010, 7, 164-175. [CrossRef]

13. Lee, D.; Bourdais, G.; Yu, G.; Robatzek, S.; Coaker, G. Phosphorylation of the plant immune regulator RPM1-INTERACTING PROTEIN4 enhances plant plasma membrane $\mathrm{H}(+)$-ATPase activity and inhibits flagellin-triggered immune responses in Arabidopsis. Plant Cell 2015, 27, 2042-2056. [CrossRef]

14. Jiang, S.S.; Yao, J.; Ma, K.W.; Zhou, H.B.; Song, J.K.; He, S.Y.; Ma, W.B. Bacterial effector activates jasmonate signaling by directly targeting JAZ transcriptional repressors. PLoS Pathog. 2013, 9, e1003715. [CrossRef] 
15. Gimenez-Ibanez, S.; Boter, M.; Fernandez-Barbero, G.; Chini, A.; Rathjen, J.P.; Solano, R. The bacterial effector HopX1 targets JAZ transcriptional repressors to activate jasmonate signaling and promote infection in Arabidopsis. PLoS Biol. 2014, 12, e1001792. [CrossRef] [PubMed]

16. Yang, L.; Teixeira, P.J.P.L.; Biswas, S.; Finkel, O.M.; He, Y.J.; Salas-Gonzalez, I.; English, M.E.; Epple, P.; Mieczkowski, P.; Dangl, J.L. Pseudomonas syringae type III effector HopBB1 promotes host transcriptional repressor degradation to regulate phytohormone responses and virulence. Cell Host Microbe 2017, 21, 156-168. [CrossRef] [PubMed]

17. Sohn, K.H.; Saucet, S.B.; Clarke, C.R.; Vinatzer, B.A.; O’Brien, H.E.; Guttman, D.S.; Jones, J.D.G. HopAS1 recognition significantly contributes to Arabidopsis nonhost resistance to Pseudomonas syringae pathogens. New Phytol. 2012, 193, 58-66. [CrossRef] [PubMed]

18. Xing, Y.; Xu, N.; Bhandari, D.D.; Lapin, D.; Sun, X.; Luo, X.; Wang, Y.; Cao, J.; Wang, H.; Coaker, G.; et al. Bacterial effector targeting of a plant iron sensor facilitates iron acquisition and pathogen colonization. Plant Cell 2021, 19, 2015-2031. [CrossRef]

19. Baltrus, D.A.; Nishimura, M.T.; Romanchuk, A.; Chang, J.H.; Mukhtar, M.S.; Cherkis, K.; Roach, J.; Grant, S.R.; Jones, C.D.; Dangl, J.L. Dynamic evolution of pathogenicity revealed by sequencing and comparative genomics of 19 Pseudomonas syringae isolates. PLoS Pathog. 2011, 7, e1002132. [CrossRef]

20. Donati, I.; Cellini, A.; Sangiorgio, D.; Vanneste, J.L.; Scortichini, M.; Balestra, G.M.; Spinelli, F. Pseudomonas syringae pv. actinidiae: Ecology, infection dynamics and disease epidemiology. Microb. Ecol. 2020, 80, 81-102. [CrossRef]

21. Scortichini, M.; Marcelletti, S.; Ferrante, P.; Petriccione, M.; Firrao, G. Pseudomonas syringae pv. actinidiae: A re-emerging, multi-faceted, pandemic pathogen. Mol. Plant Pathol. 2012, 13, 631-640. [PubMed]

22. Tyson, J.L.; Curtis, C.L.; Manning, M.A.; Rees-George, J.; Snelgar, W.P.; Blattmann, P. Systemic movement of Pseudomonas syringae pv. actinidiae in kiwifruit vines in New Zealand. N. Z. Plant Prot. 2014, 67, 41-47. [CrossRef]

23. Zhao, Z.B.; Gao, X.N.; Yang, D.H.; Huang, L.L.; Qin, H.Q.; Kang, Z.S.; Wang, N.N. Field detection of canker-causing bacteria on kiwifruit trees: Pseudomonas syringae pv. actinidiae is the major causal agent. Crop Prot. 2015, 75, 55-62. [CrossRef]

24. Gao, X.N.; Huang, Q.L.; Zhao, Z.B.; Han, Q.M.; Ke, X.W.; Qin, H.Q.; Huang, L.L. Studies on the infection, colonization, and movement of Pseudomonas syringae pv. actinidiae in kiwifruit tissues using a GFPuv-Labeled strain. PLoS ONE 2016, 11, e0151169.

25. Balestra, G.M.; Buriani, G.; Cellini, A.; Donati, I.; Mazzaglia, A.; Spinelli, F. First report of Pseudomonas syringae pv. actinidiae on kiwifruit pollen from Argentina. Plant Dis. 2018, 102, 237. [CrossRef]

26. Vleeshouwers, V.G.A.A.; Oliver, R.P. Effectors as tools in disease resistance breeding against biotrophic, hemibiotrophic, and necrotrophic plant pathogens. Mol. Plant-Microbe Interact. 2014, 27, 196-206. [CrossRef] [PubMed]

27. Choi, S.; Jayaraman, J.; Segonzac, C.; Park, H.J.; Park, H.; Han, S.W.; Sohn, K.H. Pseudomonas syringae pv. actinidiae type III effectors localized at multiple cellular compartments activate or suppress innate immune responses in Nicotiana benthamiana. Front. Plant Sci. 2017, 8, 2157. [CrossRef]

28. Jayaraman, J.; Yoon, M.; Applegate, E.R.; Stroud, E.A.; Templeton, M.D. AvrE1 and HopR1 from Pseudomonas syringae pv. actinidiae are additively required for full virulence on kiwifruit. Mol. Plant Pathol. 2020, 21, 1467-1480. [CrossRef]

29. Badel, J.L.; Nomura, K.; Bandyopadhyay, S.; Shimizu, R.; Collmer, A.; He, S.Y. Pseudomonas syringae pv. tomato DC3000 HopPtoM (CEL ORF3) is important for lesion formation but not growth in tomato and is secreted and translocated by the Hrp type III secretion system in a chaperone-dependent manner. Mol. Microbiol. 2003, 49, 1239-1251. [CrossRef]

30. Choi, S.; Prokchorchik, M.; Lee, H.; Gupta, R.; Lee, Y.; Chung, E.H.; Cho, B.; Kim, M.S.; Kim, S.T.; Sohn, K.H. Direct acetylation of a conserved threonine of RIN4 by the bacterial effector HopZ5 or AvrBsT activates RPM1-dependent immunity in Arabidopsis. Mol. Plant 2021, 14, 1951-1960. [CrossRef]

31. Choi, S.; Ayaraman, J.; Sohn, K.H. Arabidopsis thaliana SOBER1 (Suppressor of Avrbst-Elicited Resistance 1) suppresses plant immunity triggered by multiple bacterial acetyltransferase effectors. New Phytol. 2018, 219, 324-335. [CrossRef]

32. Jayaraman, J.; Choi, S.; Prokchorchik, M.; Choi, D.S.; Spiandore, A.; Rikkerink, E.H.; Templeton, M.D.; Segonzac, C.; Sohn, K.H. A bacterial acetyltransferase triggers immunity in Arabidopsis thaliana independent of hypersensitive response. Sci. Rep. 2017, 7, 3557. [CrossRef] [PubMed]

33. Yoon, M.; Rikkerink, E.H.A. Rpa1 mediates an immune response to avrRpm $1_{P s a}$ and confers resistance against Pseudomonas syringae pv. actinidiae. Plant J. 2019, 102, 688-702. [CrossRef] [PubMed]

34. Pontier, D.; Godiard, L.; Marco, Y.; Roby, D. hsr203J, a tobacco gene whose activation is rapid, highly localized and specific for incompatible plant/pathogen interactions. Plant J. 1994, 5, 507-521. [CrossRef]

35. Takahashi, Y.; Berberich, T.; Yamashita, K.; Uehara, Y.; Miyazaki, A.; Kusano, T. Identification of tobacco HIN1 and two closely related genes as spermine-responsive genes and their differential expression during the Tobacco mosaic virus -induced hypersensitive response and during leaf- and flower-senescence. Plant Mol. Biol. 2004, 54, 613-622. [CrossRef]

36. Dean, J.D.; Goodwin, P.H.; Hsiang, T. Induction of glutathione S-transferase genes of Nicotiana benthamiana following infection by Colletotrichum destructivum and C. orbiculare and involvement of one in resistance. J. Exp. Bot. 2005, 56, 1525-1533. [CrossRef] [PubMed]

37. Asai, S.; Yoshioka, H. Nitric oxide as a partner of reactive oxygen species participates in disease resistance to necrotrophic pathogen Botrytis Cinerea in Nicotiana benthamiana. Mol. Plant-Microbe Interact. 2009, 22, 619-629. [CrossRef]

38. Rodriguez, P.A.; Stam, R.; Warbroek, T.; Bos, J.I.B. Mp10 and Mp42 from the aphid species Myzus persicae trigger plant defenses in Nicotiana benthamiana through different activities. Mol. Plant-Microbe Interact. 2014, 27, 30-39. [CrossRef] 
39. Pieterse, C.M.; Van der Does, D.; Zamioudis, C.; Leon-Reyes, A.; Van Wees, S.C. Hormonal modulation of plant immunity. Annu. Rev. Cell Dev. Biol. 2012, 28, 489-521. [CrossRef]

40. Vanneste, J.L. The Scientific, Economic, and Social Impacts of the New Zealand Outbreak of Bacterial Canker of Kiwifruit (Pseudomonas syringae pv. actinidiae). Annu. Rev. Phytopathol. 2017, 55, 377-399. [CrossRef]

41. Zhao, Z.; Chen, J.; Gao, X.; Zhang, D.; Zhang, J.; Wen, J.; Qin, H.; Guo, M.; Huang, L. Comparative genomics reveal pathogenicityrelated loci in Pseudomonas syringae pv. actinidiae biovar 3. Mol. Plant Pathol. 2019, 20, 923-942. [CrossRef] [PubMed]

42. He, R.; Liu, P.; Jia, B.; Xue, S.Z.; Wang, X.J.; Hu, J.Y.; Al Shoffe, Y.; Gallipoli, L.; Mazzaglia, A.; Balestra, G.M.; et al. Genetic diversity of Pseudomonas syringae pv. actinidiae strains from different geographic regions in China. Phytopathology 2019, 109, 347-357. [CrossRef] [PubMed]

43. Sawada, H.; Fujikawa, T. Genetic diversity of Pseudomonas syringae pv. actinidiae, pathogen of kiwifruit bacterial canker. Plant Pathol. 2019, 68, 1235-1248. [CrossRef]

44. Tzelepis, G.; Dolfors, F.; Holmquist, L.; Dixelius, C. Plant mitochondria and chloroplasts are targeted by the Rhizoctonia solani RsCRP1 effector. Biochem. Biophys. Res. Commun. 2021, 544, 86-90. [CrossRef] [PubMed]

45. Jelenska, J.; Yao, N.; Vinatzer, B.A.; Wright, C.M.; Brodsky, J.L.; Greenberg, J.T. A J domain virulence effector of Pseudomonas syringae remodels host chloroplasts and suppresses defenses. Curr. Biol. 2007, 17, 499-508. [CrossRef] [PubMed]

46. Rodriguez-Herva, J.J.; Gonzalez-Melendi, P.; Cuartas-Lanza, R.; Antunez-Lamas, M.; Rio-Alvarez, I.; Li, Z.; Lopez-Torrejon, G.; Diaz, I.; del Pozo, J.C.; Chakravarthy, S.; et al. A bacterial cysteine protease effector protein interferes with photosynthesis to suppress plant innate immune responses. Cell. Microbiol. 2012, 14, 669-681. [CrossRef]

47. Tang, L.; Yang, G.; Ma, M.; Liu, X.; Li, B.; Xie, J.; Fu, Y.; Chen, T.; Yu, Y.; Chen, W.; et al. An effector of a necrotrophic fungal pathogen targets the calcium-sensing receptor in chloroplasts to inhibit host resistance. Mol. Plant Pathol. 2020, 21, 686-701. [CrossRef]

48. Nomura, H.; Komori, T.; Uemura, S.; Kanda, Y.; Shimotani, K.; Nakai, K.; Furuichi, T.; Takebayashi, K.; Sugimoto, T.; Sano, S.; et al. Chloroplast-mediated activation of plant immune signalling in Arabidopsis. Nat. Commun. 2012, 3, 926. [CrossRef]

49. Han, S.; Tang, R.; Anderson, L.K.; Woerner, T.E.; Pei, Z.M. A cell surface receptor mediates extracellular $\mathrm{Ca}\left({ }^{2+}\right)$ sensing in guard cells. Nature 2003, 425, 196-200. [CrossRef]

50. Tang, R.H.; Han, S.; Zheng, H.; Cook, C.W.; Choi, C.S.; Woerner, T.E.; Jackson, R.B.; Pei, Z.M. Coupling diurnal cytosolic Ca ${ }^{2+}$ oscillations to the CAS-IP3 pathway in Arabidopsis. Science 2007, 315, 1423-1426. [CrossRef]

51. Weinl, S.; Held, K.; Schlucking, K.; Steinhorst, L.; Kuhlgert, S.; Hippler, M.; Kudla, J. A plastid protein crucial for Ca ${ }^{2+}$-regulated stomatal responses. New Phytol. 2008, 179, 675-686. [CrossRef]

52. Huang, S.S.; Chen, J.; Dong, X.J.; Patton, J.; Pei, Z.M.; Zheng, H.L. Calcium and calcium receptor CAS promote Arabidopsis thaliana de-etiolation. Physiol. Plantarum. 2012, 144, 73-82. [CrossRef] [PubMed]

53. Wang, W.H.; Yi, X.Q.; Han, A.D.; Liu, T.W.; Chen, J.; Wu, F.H.; Dong, X.J.; He, J.X.; Pei, Z.M.; Zheng, H.L. Calcium-sensing receptor regulates stomatal closure through hydrogen peroxide and nitric oxide in response to extracellular calcium in Arabidopsis. J. Exp. Bot. 2012, 63, 177-190. [CrossRef] [PubMed]

54. Medina-Puche, L.; Kim, C.; Lozano-Duran, R.; Dogra, V. Protocol for evaluating protein relocalization from the plasma membrane to chloroplasts. STAR Protoc. 2021, 14, 100816. [CrossRef]

55. Medina-Puche, L.; Tan, H.; Dogra, V.; Wu, M.; Rosas-Diaz, T.; Wang, L.; Ding, X.; Zhang, D.; Fu, X.; Kim, C.; et al. A defense pathway linking plasma membrane and chloroplasts and co-opted by pathogens. Cell 2020, 182, 1109-1124 e25. [CrossRef] [PubMed]

56. Wang, K.; Kang, L.; Anand, A.; Lazarovits, G.; Mysore, K.S. Monitoring in planta bacterial infection at both cellular and whole-plant levels using the green fluorescent protein variant GFPuv. New Phytol. 2007, 174, 212-223. [CrossRef]

57. Kvitko, B.H.; Collmer, A. Construction of Pseudomonas syringae pv. tomato DC3000 mutant and polymutant strains. Methods Mol. Biol. 2011, 712, 109-128.

58. Yu, X.; Feng, B.; He, P.; Shan, L. From chaos to harmony: Responses and signaling upon microbial pattern recognition. Annu. Rev. Phytopathol. 2017, 55, 109-137. [CrossRef]

59. Golpayegani, A.; Nodehi, R.N.; Rezaei, F.; Alimohammadi, M.; Douraghi, M. Real-time polymerase chain reaction assays for rapid detection and virulence evaluation of the environmental Pseudomonas aeruginosa isolates. Mol. Biol. Rep. 2019, 46, 4049-4061. [CrossRef] [PubMed]

60. Jiang, N.; Lyu, Q.; Han, S.; Xu, X.; Walcott, R.R.; Li, J.; Luo, L. Evaluation of suitable reference genes for normalization of quantitative reverse transcription PCR analyses in Clavibacter michiganensis. MicrobiologyOpen 2019, 8, e928. [CrossRef]

61. Sainsbury, F.; Lomonossoff, G.P. Extremely high-level and rapid transient protein production in plants without the use of viral replication. Plant Physiol. 2008, 148, 1212-1218. [CrossRef] [PubMed]

62. Schmittgen, T.D.; Livak, K.J. Analyzing real-time PCR data by the comparative C-T method. Nat. Protoc. 2008, 3, 1101-1108. [CrossRef] [PubMed]

63. Nie, J.J.; Yin, Z.Y.; Li, Z.P.; Wu, Y.X.; Huang, L.L. A small cysteine-rich protein from two kingdoms of microbes is recognized as a novel pathogen-associated molecular pattern. New Phytol. 2019, 222, 995-1011. [CrossRef]

64. Qi, M.; Link, T.I.; Muller, M.; Hirschburger, D.; Pudake, R.N.; Pedley, K.F.; Braun, E.; Voegele, R.T.; Baum, T.J.; Whitham, S.A. A small cysteine-rich protein from the Asian soybean rust fungus, Phakopsora pachyrhizi, suppresses plant immunity. PLoS Pathog. 2016, 12, e1005827. [CrossRef] [PubMed] 